\title{
Research Article \\ Decreased Serum NCAM Levels Associated with Cognitive Impairment in Vascular Dementia
}

\author{
Jun Zhao, ${ }^{1}$ Wei Lu, ${ }^{2}$ Junshan $\mathrm{Li}^{3}{ }^{3}$ Lei Liu, ${ }^{4}$ and Xiumin Zhao $\mathbb{D D}^{4}$ \\ ${ }^{1}$ Department of Neurology, Qingpu Branch of Zhongshan Hospital, Fudan University, Shanghai 201700, China \\ ${ }^{2}$ Department of Intensive Rehabilitation, Shandong Provincial Third Hospital, Cheeloo College of Medicine, Shandong University, \\ Jinan, Shandong Province 250000, China \\ ${ }^{3}$ Department of Gastroenterology, Shandong Provincial Third Hospital, Cheeloo College of Medicine, Shandong University, Jinan, \\ Shandong Province 250000, China \\ ${ }^{4}$ Department of Neurology, Shandong Provincial Third Hospital, Cheeloo College of Medicine, Shandong University, Jinan, \\ Shandong Province 250000, China
}

Correspondence should be addressed to Xiumin Zhao; rice365@sina.com

Received 5 August 2021; Revised 17 August 2021; Accepted 19 August 2021; Published 31 August 2021

Academic Editor: Yuzhen Xu

Copyright (c) 2021 Jun Zhao et al. This is an open access article distributed under the Creative Commons Attribution License, which permits unrestricted use, distribution, and reproduction in any medium, provided the original work is properly cited.

\begin{abstract}
Objective. Neural cell adhesion molecule (NCAM), a glycoprotein widely distributed in the brain, has recently been shown to regulate neuroplasticity. However, the role of NCAM in vascular dementia (VaD) is still unclear. The purpose of this study is to determine whether NCAM is involved in the course of VaD. Methods. Continuous recruitment of VaD patients and control population to join this study. Doctors or nurses are responsible for collecting their clinical characteristics including age, gender, formal education, heart rate, supine systolic blood pressure, supine diastolic blood pressure, fasting glucose, high-density lipoprotein, and low-density lipoprotein. Each participant received the Montreal Cognitive Assessment (MoCA) scale after being enrolled in the group. At the same time, their peripheral blood was collected, and their serum NCAM levels were measured by enzyme-linked immunosorbent assay (ELISA). Results. $98 \mathrm{VaD}$ patients and 83 age- and sex-matched controls were enrolled. There was no significant statistical difference between the VaD group and the control group in terms of the comparison of clinical characteristics $(p>0.05)$. The MoCA score of $\mathrm{VaD}$ patients was significantly lower than that of the controls $(27.9 \pm 1.4$ vs. $23.0 \pm 2.1$ points, $p<0.001)$. In addition, the circulating NCAM level of VaD patients was also significantly lower than that of controls $(21.7 \pm 3.8$ vs. $17.6 \pm 4.2 \mathrm{ng} / \mathrm{mL}, p<0.001)$. The circulating NCAM level of $\mathrm{VaD}$ patients was significantly positively correlated with MoCA score $(r=0.285, p=0.026)$. After adjusting for clinical characteristics, circulating NCAM levels are still an independent pathogenic factor of VaD (regression coefficient $=0.223, p=0.034)$. Conclusions. $\mathrm{VaD}$ patients have low circulating NCAM levels, which can be used as a potential predictor of $\mathrm{VaD}$.
\end{abstract}

\section{Introduction}

Vascular dementia $(\mathrm{VaD})$ is a general term describing brain damage caused by cerebral blood flow disorders, which can cause problems in reasoning, judgment, learning, planning, memory, and other thinking processes [1-3]. About 15$20 \%$ of people with dementia will develop $\mathrm{VaD}$. It is the second most common dementia among people over 65 [4]. As people's lifestyle changes and life pressure increases, the prevalence of $\mathrm{VaD}$ remains high. The latest epidemiological studies show that the number of people diagnosed with dementia will rapidly increase to 131 million by 2050 [5, 6]. Hypertension, diabetes, smoking, hyperlipidemia, and arrhythmia are common causes of $\mathrm{VaD}[7,8]$. However, current treatments based on controlling risk factors cannot fundamentally alleviate $\mathrm{VaD}$.

Wilson discovered the phenomenon of cell adhesion in 1907, and the neural cell adhesion molecule (NCAM), a special cell adhesion molecule, was discovered by Jørgensen and Bock in 1974, and it was originally named to synaptic 
membrane protein D2 $[9,10]$. NCAM is encoded by a gene located on chromosome 11. Due to alternative splicing, it is divided into three main subtypes according to its molecular weight: NCAM-120, NCAM-140, and NCAM-180 [11, 12]. The intracellular parts of these three NCAM subtypes are different, so they can show different expression patterns and functions [13]. NCAM, also known as CD56, belongs to the immunoglobulin superfamily and is a glycoprotein mainly expressed in neurons and glial cells [14]. NCAM plays an important role in neurodevelopment, neuromigration, synaptic growth, and neuroplasticity and affects the formation of learning and memory [15].

In the decades after the discovery of NCAM, its role in a variety of neuropsychological and neurodegenerative diseases has been widely reported. NCAM has also been isolated and identified, and its mimic peptide is also considered a neuroprotective agent. However, the relationship between NCAM and $\mathrm{VaD}$, the second largest type of dementia, is still unclear. The purpose of this study is to explore the relationship between NCAM and the cognitive function of $\mathrm{VaD}$ patients.

\section{Methods}

2.1. Study Subjects. From January 2019 to December 2020, $\mathrm{VaD}$ patients treated in the outpatient and ward of Shandong Provincial Third Hospital were registered. The diagnosis of $\mathrm{VaD}$ patients is based on the Diagnostic and Statistical Manual of Mental Disorders V (DSM-5) and National Institute for Neurological Disorders and Stroke (NINDSAIREN), made by experienced neurologists. All subjects in the group were examined by computed tomography (CT) or magnetic resonance imaging (MRI). The inclusion criteria are as follows: (1) age over 50 years old; (2) no intracranial hemorrhage, deformity, or tumor in imaging examination; and (3) meet the diagnostic criteria of $\mathrm{VaD}$. The exclusion criteria are as follows: (1) other causes of dementia, such as Alzheimer's disease (AD) and Lewy body dementia (DLB); (2) acute phase of new stroke; (3) history of previous head trauma or surgery; (4) severe psychological or mental illness; (5) dependence on drugs, alcohol, or heroin; (6) taking nootropics such as donepezil, rivastigmine, and sodium oligomannate (GV971); (7) unable to complete neuropsychological tests; and (8) individuals or the family refused to sign the informed consent. In addition, healthy volunteers without cognitive impairment were recruited as controls. All participants or their family members were informed of this study and agreed to participate in the study. The protocol of this study complies with the Declaration of Helsinki and has been approved by the local ethics committee. All research methods are strictly implemented in accordance with the relevant guidelines.

2.2. Clinical Characteristics Collection. After the study subjects were enrolled in the group, specialized medical staff were responsible for collecting their clinical characteristics. The collected clinical characteristics include age, gender, formal education, heart rate, supine systolic blood pressure, supine diastolic blood pressure, fasting glucose, high- density lipoprotein, and low-density lipoprotein. The age, gender, and formal education information is provided by the participant or a familiar caregiver. The remaining biochemical indicators are tested in the hospital laboratory according to standardized biochemical methods. All clinical characteristics were collected within 48 hours after enrollment and carefully recorded for analysis.

2.3. Cognitive Function Evaluation. The Montreal Cognitive Assessment (MoCA) is widely used as a screening test for highly educated elderly people with mild cognitive impairment and is generally considered to be suitable for subjects who have been educated for more than 13 years. Due to the low average education level of the elderly in China, our study adopted the MoCA basic test (MoCA-B). The purpose of revising the MoCA test is to make it suitable for individuals with lower education levels. "MoCA-B" is a slightly modified version of language and culture after review and approval by the creator of MoCA, calculation, concentration, executive function, conceptual thinking, and visual perception [16]. Like MoCA, the total score of the MoCA-B scale is 30 points, and a score lower than 26 points is considered to have cognitive impairment [17]. MoCA-B is online for free use by clinicians Dr. Ziad Nasreddine. It includes nine cognitive dimensions: language, direction, attention, and memory (http://www.mocatest.org/).

2.4. Biomarker Assessment. The circulating levels of NCAM are detected by enzyme-linked immunosorbent assay (ELISA). Commercial ELISA detection reagents were purchased from MyBioSource ELISA kit (MyBiosource, Inc., San Diego, CA, USA). The detection of this reagent adopts the sandwich method, and its detection sensitivity is $1.0 \mathrm{ng} / \mathrm{mL}$, and the detection range is $3.12 \mathrm{ng} / \mathrm{mL}$ $100 \mathrm{ng} / \mathrm{mL}$. The peripheral blood of the participants was collected, left at room temperature for 20 minutes, and then centrifuged at $1000 \mathrm{~g}$ for about 20 minutes. Carefully collect the supernatant to obtain the serum, and test it immediately or store the sample at $-80^{\circ} \mathrm{C}$ to avoid repeated freezing and thawing [18]. The experiment sets up standard wells, sample wells, and blank/control wells, and each sample is measured repeatedly 3 times. The detection method is based on the product specification.

2.5. Statistical Methods. The statistics of measurement data are expressed as mean \pm standard deviation, and the statistics of count data are expressed as $n$ (\%). Two independent samples $t$-test or $X 2$ test was used for the uniformity of baseline data in the two groups. The bivariate Pearson and Spearman correlation coefficient was used to assess the correlation between the MoCA score and clinical characteristics. Multivariate regression analysis was used to evaluate the causal relationship between serum circulating NCAM levels and $\mathrm{VaD}$ after adjusting for clinical characteristics. All analyses are done with SPSS software package (IBM SPSS Statistics for Windows, Version 20.0). All hypothesis tests are significant with a two-tailed $p$ value $<0.05$. 


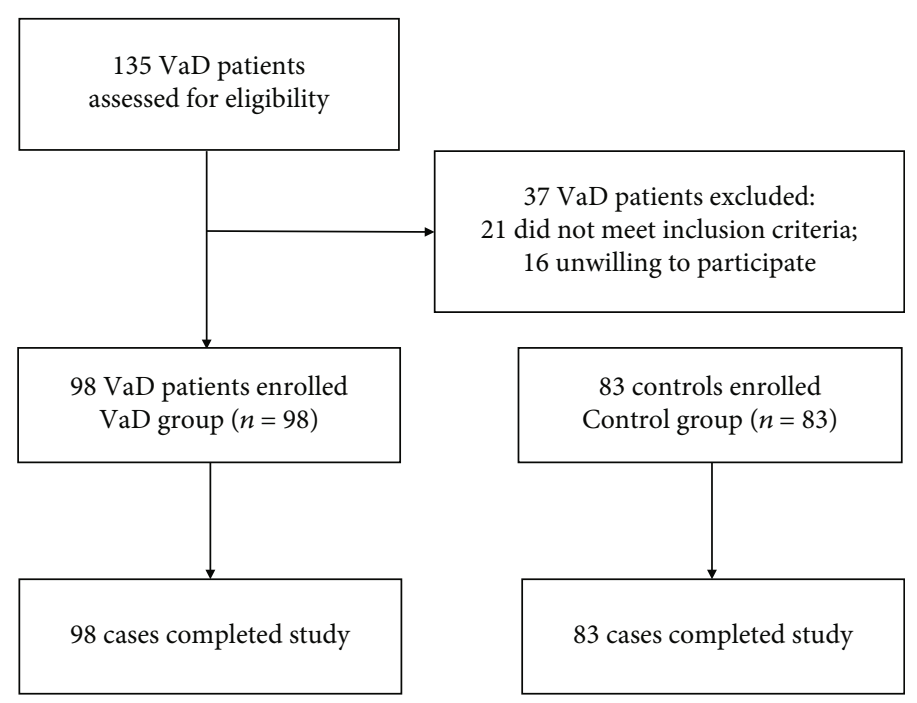

FIGURE 1: Flow diagram of the study. VaD: vascular dementia.

\section{Results}

3.1. Clinical Characteristics of All Participants. From January 2019 to December 2020, $135 \mathrm{VaD}$ patients were screened in the outpatient and ward of Shandong Provincial Third Hospital. After screening, 37 people were excluded, of which 21 did not meet the inclusion criteria, and the remaining 16 refused to participate. This leaves $98 \mathrm{VaD}$ participants who meet all the inclusion criteria, and they are assigned to the $\mathrm{VaD}$ group $(n=98)$. We enrolled 83 people without cognitive impairment as the control group $(n=83)$. In the end, all participants in the group completed the study and provided data for analysis. The flow diagram of the study is shown in Figure 1.

The clinical characteristics of the two groups are similar, and there is no significant difference between them $(p>0.05$ ). These similar clinical characteristics include age, gender, formal education, heart rate, supine systolic blood pressure, supine diastolic blood pressure, fasting glucose, highdensity lipoprotein, and low-density lipoprotein, as shown in Table 1.

However, the MoCA score of $\mathrm{VaD}$ patients was significantly lower than that of the control $(27.9 \pm 1.4$ vs. $23.0 \pm$ 2.1 points, $p<0.001)$. In addition, the circulating NCAM level of $\mathrm{VaD}$ patients was also significantly lower than that of controls $(21.7 \pm 3.8$ vs. $17.6 \pm 4.2 \mathrm{ng} / \mathrm{mL}, p<0.001)$. The significant differences in MoCA scores and circulating serum NCAM levels between the two groups are shown in Figure 2 .

3.2. Bivariate Correlation Analysis. To evaluate the correlation between clinical characteristics and cognitive function, we did a bivariate correlation analysis. As shown in Table 2, the bivariate analysis between age, gender, formal education, heart rate, supine systolic blood pressure, supine diastolic blood pressure, fasting glucose, high-density lipoprotein, low-density lipoprotein, and MoCA score shows that they are not significantly related. However, there was a
TABLE 1: Clinical characteristics in controls and $\mathrm{VaD}$ patients.

\begin{tabular}{lccc}
\hline & $\begin{array}{c}\text { Controls } \\
(n=83)\end{array}$ & $\begin{array}{c}\text { VaD } \\
(n=98)\end{array}$ & $\begin{array}{c}p \\
\text { value }\end{array}$ \\
\hline Age (years) & $67.4 \pm 7.9$ & $67.8 \pm 8.1$ & 0.738 \\
Male gender ( $n$ \%) & $56(67.5)$ & $70(71.4)$ & 0.564 \\
Formal education (years) & $10.6 \pm 3.2$ & $10.3 \pm 3.4$ & 0.544 \\
Heart rate (bpm) & $71.5 \pm 12.7$ & $70.8 \pm 12.0$ & 0.704 \\
$\begin{array}{l}\text { Supine systolic BP } \\
\text { (mmHg) }\end{array}$ & $138.2 \pm 13.6$ & $137.7 \pm 13.8$ & 0.807 \\
$\begin{array}{l}\text { Supine diastolic BP } \\
\text { (mmHg) }\end{array}$ & $81.4 \pm 10.9$ & $81.9 \pm 11.1$ & 0.761 \\
FBG (mmol/L) & $6.5 \pm 0.4$ & $6.6 \pm 0.3$ & 0.057 \\
HDL cholesterol (mmol/ & $1.4 \pm 0.5$ & $1.3 \pm 0.3$ & 0.099 \\
L) & $2.7 \pm 0.4$ & $2.8 \pm 0.5$ & 0.144 \\
LDL cholesterol (mmol/L) & $27.9 \pm 1.4$ & $23.0 \pm 2.1$ & $<0.001$ \\
MoCA (points) & $21.7 \pm 3.8$ & $17.6 \pm 4.2$ & $<0.001$ \\
\hline NCAM (ng/mL) & &
\end{tabular}

VaD: vascular dementia; BP: blood pressure; FBG: fasting blood glucose; HDL: high-density lipoprotein; LDL: low-density lipoprotein; MoCA: Montreal Cognitive Assessment; NCAM: neural cell adhesion molecule.

significant positive correlation between circulating NCAM concentration and MoCA score $(r=0.258, p=0.026)$.

3.3. Multivariate Regression Analysis. In order to further verify the correlation between clinical characteristics and cognitive function, we did a multivariate regression analysis to eliminate the influence of confounding factors on the conclusion as much as possible. After correction for age, gender, formal education, heart rate, supine systolic blood pressure, supine diastolic blood pressure, fasting glucose, highdensity lipoprotein, and low-density lipoprotein, circulating NCAM levels can still significantly affect the cognitive function of $\mathrm{VaD}$ patients (regression coefficient $=0.223, p=$ 0.034 ). The results of the multivariate analysis are summarized in Table 3. 


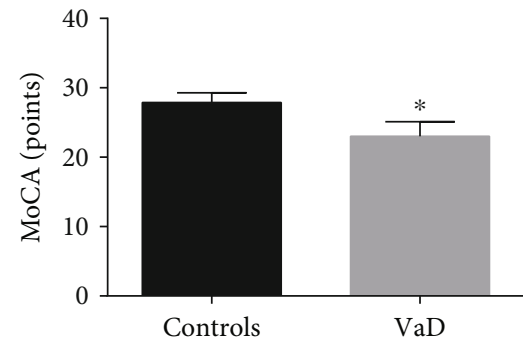

(a)

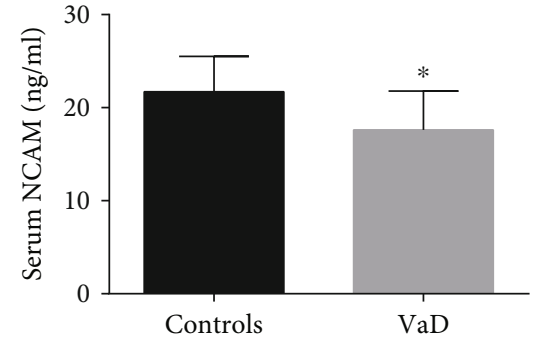

(b)

FIgure 2: The MoCA scores and circulating levels of NCAM in all subjects: (a) MoCA scores; (b) circulating levels of NCAM. Compared with the controls, ${ }^{*} p<0.05$.

TABLE 2: Correlation between clinical characteristics and MoCA in patients with $\mathrm{VaD}$.

\begin{tabular}{lcc}
\hline & $r$ & $p$ value \\
\hline Age (years) & -0.319 & 0.362 \\
Male gender $(n, \%)$ & -0.283 & 0.445 \\
Formal education (years) & 0.472 & 0.236 \\
Heart rate (bpm) & 0.271 & 0.637 \\
Supine systolic BP (mmHg) & 0.118 & 0.302 \\
Supine diastolic BP (mmHg) & 0.184 & 0.143 \\
FBG (mmol/L) & -0.362 & 0.593 \\
HDL cholesterol (mmol/L) & 0.299 & 0.108 \\
LDL cholesterol (mmol/L) & -0.280 & 0.094 \\
NCAM (ng/mL) & 0.285 & 0.026 \\
\hline
\end{tabular}

MoCA: Montreal Cognitive Assessment; VaD: vascular dementia; BP: blood pressure; FBG: fasting blood glucose; HDL: high-density lipoprotein; LDL: low-density lipoprotein; NCAM: neural cell adhesion molecule.

\section{Discussion}

In our study, we tested the differences in circulating NCAM levels and cognitive function between $\mathrm{VaD}$ patients and control groups. The results showed that the MoCA score and circulating NCAM levels in $\mathrm{VaD}$ patients were significantly reduced. These results suggest that low circulating NCAM levels may be related to the pathogenesis of $\mathrm{VaD}$. In order to evaluate the possible link between $\mathrm{VaD}$ and circulating NCAM level, we did a correlation and regression analysis between circulating NCAM level and MoCA score. The results showed that even after adjusting for confounding factors, there was still a significant positive correlation between MoCA scores and circulating NCAM levels. As far as we know, there are currently limited papers on the correlation between circulating NCAM levels and VaD.

Cell adhesion molecule (CAM) is a ligand that participates in cell-cell recognition in vertebrates and can participate in pathophysiological processes such as the selective binding of axons, the selection of synaptic targets, or cell separation [19]. In the nervous system, many membrane glycoproteins or transmembrane proteins are identified as CAM proteins. NCAM is the first CAM protein identified as mainly expressed in the nervous system and can affect various cellular events in the nervous system during development or after maturation [20]. There are three main forms
TABLE 3: Multivariable analyses of clinical characteristics and MoCA in patients with $\mathrm{VaD}$.

\begin{tabular}{lccc}
\hline & $\begin{array}{c}\text { Regression } \\
\text { coefficient }\end{array}$ & $95 \% \mathrm{CI}$ & $\begin{array}{c}p \\
\text { value }\end{array}$ \\
\hline Age (years) & 0.291 & $0.216-$ & 0.221 \\
& & 1.431 & \\
Male gender ( $n$, \%) & 0.265 & $0.178-$ & 0.143 \\
& & 1.257 & \\
Formal education (years) & 0.316 & $0.109-$ & 0.436 \\
& & 1.345 & \\
Heart rate (bpm) & 0.117 & $0.036-$ & 0.380 \\
Supine systolic BP & & 1.134 & \\
(mmHg) & 0.138 & $0.053-$ & 0.529 \\
Supine diastolic BP & & 1.179 & \\
(mmHg) & 0.147 & $0.072-$ & 0.315 \\
FBG (mmol/L) & & 1.196 & \\
& 0.330 & $0.128-$ & 0.137 \\
HDL cholesterol (mmol/ & \multirow{3}{3}{1.310} & \\
L) & 0.246 & $0.167-$ & 0.412 \\
LDL cholesterol (mmol/ & \multirow{3}{*}{1.183} & \\
L) & 0.209 & $0.097-$ & 0.318 \\
NCAM (ng/mL) & \multirow{3}{*}{1.202} & \\
& 0.223 & $0.101-$ & 0.034 \\
\hline
\end{tabular}

MoCA: Montreal Cognitive Assessment; VaD: vascular dementia; BP: blood pressure; FBG: fasting blood glucose; HDL: high-density lipoprotein; LDL: low-density lipoprotein; NCAM: neural cell adhesion molecule.

of NCAM. Among them, the large molecular weight is transmembrane proteins, while the small molecular weight NCAM-120 is attached to the membrane through glycosylphosphatidylinositol anchors [21]. NCAM can become soluble NCAM after being digested by restriction enzymes, which is widely present in the brain, cerebrospinal fluid (CSF), and plasma [22]. The mechanism of NCAM's biological effects in the body is complicated. On the one hand, NCAM mediates cell adhesion through homotypic and heterotypic interactions, thereby activating transmembrane signals and triggering calcium influx [23]. On the other hand, NCAM mediates the adhesion of cells to other cells or extracellular matrix and affects cell migration, neurite extension, and synapse formation by activating intracellular signaling pathways [24]. In addition, polysialic acid is thought to affect 
its biological functions in vivo and in vitro by regulating the cell adhesion activity of NCAM [25].

NCAM may play an important role in a series of neurological diseases. Gnanapavan's research team compared the differences in CSF NCAM levels in control populations with benign intracranial hypertension, multiple sclerosis, cases of $\mathrm{AD}$, motor neuron disease, and meningitis and found that its levels in multiple sclerosis, $\mathrm{AD}$, and meningitis are reduced [26]. The autopsy results of patients with refractory temporal lobe epilepsy showed that the expression of highly salivated NCAM in the hippocampus and entorhinal cortex increased, indicating that NCAM is involved in the remodeling of neuronal circuits in patients with epilepsy [27]. In the hippocampus of patients with schizophrenia, PSA-NCAM immunoreactivity is reduced, and there are more embryonic structures of NCAM, indicating that the plasticity of NCAM in the brain has changed, which further affects neurodevelopment [28]. Interestingly, NCAM is undetectable in normal muscle fibers, but it is abundantly expressed in denervated and regenerated muscle fibers, suggesting that NCAM can participate in the pathological process of neuromyopathy by promoting nerve regeneration [29, 30]. In addition, the relationship between CAM and stroke has long been discovered, but clinical trials for CAM targeted therapy have not been successful, indicating that the pathogenic mechanism of CAM has not been fully elucidated [31].

Accumulated evidence indicates that NCAM is a key regulator of synaptic plasticity, which can affect learning, memory, and cognitive function [32]. Indian scholars have found that the expression of neuroplasticity marker NCAM is increased in patients with bipolar disorder and is related to the severity of the disease [33]. German researchers confirmed that NCAM regulates synaptic plasticity and learning ability by inhibiting the signal transduction of NMDA receptors containing GluN2B [34]. A recent multinational joint study showed that NCAM plays an important neuroprotective role in the associative memory of C. elegans and humans, which proposes new therapeutic targets for memory-related diseases [35]. The effect of NCAM on neuroplasticity has been verified in cognitive impairmentrelated diseases or models. Scientists from Denmark found that an NCAM-derived peptide reduces the characteristic neuropathological changes and cognitive impairment induced by A $\beta 25-35$ [36]. Clinical studies in Argentina have also shown that NCAM may be involved in the pathogenesis of $\mathrm{AD}$ disease and can be used as a differential diagnostic marker for the disease [37]. However, the role of NCAM in $\mathrm{VaD}$ has not been reported yet.

The advantage of our research is that we reported for the first time that NCAM is involved in the pathogenesis of $\mathrm{VaD}$. However, our research also has some limitations. First of all, our study is a small cross-sectional study, lacking intervention studies for diagnostic targets. Secondly, VaD patients have different disease durations, which makes it impossible for us to track the dynamic changes of NCAM in the course of $\mathrm{VaD}$ disease. Finally, the etiology of $\mathrm{VaD}$ is different, which may also cause NCAM to be unable to accurately interpret the conclusions.

\section{Conclusions}

In summary, the highlight of our study is the discovery that low levels of circulating NCAM are closely related to the cognitive function of $\mathrm{VaD}$. The level of circulating NCAM may be an indicator of the cognitive function of $\mathrm{VaD}$ patients. It is worthwhile for us to do clinical efficacy trials for intervention in NCAM in the future. The relationship between circulating NCAM levels and $\mathrm{VaD}$ cognitive function may provide a new treatment portal for $\mathrm{VaD}$.

\section{Data Availability}

The data used to support the findings of this study are available from the corresponding author upon request.

\section{Conflicts of Interest}

All the authors declare no conflict of interests.

\section{Authors' Contributions}

Jun Zhao and Wei Lu are co-first authors and contributed equally to this work.

\section{Acknowledgments}

This research work was supported by a grant from the Natural Science Foundation of Shandong Province, China (ZR2020MH135).

\section{References}

[1] J. T. O'Brien and A. Thomas, "Vascular dementia," Lancet (London, England), vol. 386, no. 10004, pp. 1698-1706, 2015.

[2] Q. Wang, Y. Xu, C. Qi, A. Liu, and Y. Zhao, "Association study of serum soluble TREM2 with vascular dementia in Chinese Han population," The International Journal of Neuroscience, vol. 130, no. 7, pp. 708-712, 2020.

[3] X. Wang, F. Zhang, W. Ma, D. Feng, J. Zhang, and J. Xu, "Increased levels of serum neuregulin 1 associated with cognitive impairment in vascular dementia," BioMed Research International, vol. 2020, Article ID 6683747, 5 pages, 2020.

[4] G. C. Román, "Facts, myths, and controversies in vascular dementia," Journal of the Neurological Sciences, vol. 226, no. 1-2, pp. 49-52, 2004.

[5] Y. Xu, Q. Wang, Z. Qu, J. Yang, X. Zhang, and Y. Zhao, "Protective effect of hyperbaric oxygen therapy on cognitive function in patients with vascular dementia," Cell Transplantation, vol. 28, no. 8, pp. 1071-1075, 2019.

[6] J. Li, S. Li, Y. Song et al., "Association of serum FAM19A5 with cognitive impairment in vascular dementia," Disease Markers, vol. 2020, Article ID 8895900, 5 pages, 2020.

[7] F. J. Wolters and M. A. Ikram, "Epidemiology of vascular dementia," Arteriosclerosis, Thrombosis, and Vascular Biology, vol. 39, no. 8, pp. 1542-1549, 2019.

[8] L. Jia, M. Quan, Y. Fu et al., "Dementia in China: epidemiology, clinical management, and research advances," The Lancet Neurology, vol. 19, no. 1, pp. 81-92, 2020.

[9] D. Nathaniel Clarke, C. J. Lowe, and W. James Nelson, "The cadherin-catenin complex is necessary for cell adhesion and 
embryogenesis in Nematostella vectensis," Developmental Biology, vol. 447, no. 2, pp. 170-181, 2019.

[10] O. Jørgensen and E. Bock, "Brain specific synaptosomal membrane proteins demonstrated by crossed immunoelectrophoresis," Journal of Neurochemistry, vol. 23, no. 4, pp. 879-880, 1974.

[11] M. Axelsson, N. Dubuisson, L. Novakova et al., "Cerebrospinal fluid NCAM levels are modulated by disease-modifying therapies," Acta Neurologica Scandinavica, vol. 139, no. 5, pp. 422427, 2019.

[12] M. Životić, B. Tampe, G. Müller et al., "Modulation of NCAM/FGFR1 signaling suppresses EMT program in human proximal tubular epithelial cells," PLoS One, vol. 13, no. 11, article e0206786, 2018.

[13] B. Büttner and R. Horstkorte, "Intracelluar ligands of NCAM," Structure and Function of the Neural Cell Adhesion Molecule NCAM, vol. 663, pp. 55-66, 2010.

[14] J. T. Gunesch, A. L. Dixon, T. A. Ebrahim et al., "CD56 regulates human NK cell cytotoxicity through Pyk2," eLife, vol. 9, article e57346, 2020.

[15] E. Gascon, L. Vutskits, and J. Z. Kiss, "Polysialic acid-neural cell adhesion molecule in brain plasticity: from synapses to integration of new neurons," Brain Research Reviews, vol. 56, no. 1, pp. 101-118, 2007.

[16] L. Huang, K. L. Chen, B. Y. Lin et al., "Chinese version of Montreal Cognitive Assessment Basic for discrimination among different severities of Alzheimer's disease," Neuropsychiatric Disease and Treatment, vol. 14, pp. 2133-2140, 2018.

[17] Y. Xu, Q. Wang, Y. Liu, R. Cui, and Y. Zhao, "Is Helicobacter pylori infection a critical risk factor for vascular dementia?," The International Journal of Neuroscience, vol. 126, no. 10, pp. 899-903, 2016.

[18] J. Zhang, L. Tang, J. Hu, Y. Wang, and Y. Xu, "Uric acid is associated with cognitive impairment in the elderly patients receiving maintenance hemodialysis-a two-center study," Brain and behavior, vol. 10, no. 3, article e01542, 2020.

[19] Y. Zhang, J. Yeh, P. M. Richardson, and X. Bo, "Cell adhesion molecules of the immunoglobulin superfamily in axonal regeneration and neural repair," Restorative Neurology and Neuroscience, vol. 26, no. 2-3, pp. 81-96, 2008.

[20] T. Seki and Y. Arai, "Distribution and possible roles of the highly polysialylated neural cell adhesion molecule (NCAM$\mathrm{H}$ ) in the developing and adult central nervous system," Neuroscience Research, vol. 17, no. 4, pp. 265-290, 1993.

[21] L. Li, H. Chen, M. Wang et al., "NCAM-140 translocation into lipid rafts mediates the neuroprotective effects of GDNF," Molecular Neurobiology, vol. 54, no. 4, pp. 2739-2751, 2017.

[22] L. Rønn, B. Hartz, and E. Bock, "The neural cell adhesion molecule (NCAM) in development and plasticity of the nervous system," Experimental Gerontology, vol. 33, no. 7-8, pp. 853864, 1998.

[23] R. Juliano, "SIGNALTRANSDUCTION BYCELLADHESIONRECEPTORS and THECYTOSKELETON: functions of integrins, cadherins, selectins, and immunoglobulinsuperfamily members," Annual Review of Pharmacology and Toxicology, vol. 42, no. 1, pp. 283-323, 2002.

[24] T. Wang, S. Hamilla, M. Cam, H. Aranda-Espinoza, and S. Mili, "Extracellular matrix stiffness and cell contractility control RNA localization to promote cell migration," Nature Communications, vol. 8, no. 1, pp. 896-916, 2017.
[25] K. Angata and M. Fukuda, "Polysialyltransferases: major players in polysialic acid synthesis on the neural cell adhesion molecule," Biochimie, vol. 85, no. 1-2, pp. 195-206, 2003.

[26] S. Gnanapavan, D. Grant, E. Illes-Toth, N. Lakdawala, G. Keir, and G. Giovannoni, "Neural cell adhesion molecule - description of a CSF ELISA method and evidence of reduced levels in selected neurological disorders," Journal of Neuroimmunology, vol. 225, no. 1-2, pp. 118-122, 2010.

[27] M. Mikkonen, H. Soininen, R. Kälviäinen et al., "Remodeling of neuronal circuitries in human temporal lobe epilepsy: increased expression of highly polysialylated neural cell adhesion molecule in the hippocampus and the entorhinal cortex," Annals of Neurology, vol. 44, no. 6, pp. 923-934, 1998.

[28] D. Barbeau, J. J. Liang, Y. Robitalille, R. Quirion, and L. K. Srivastava, "Decreased expression of the embryonic form of the neural cell adhesion molecule in schizophrenic brains," Proceedings of the National Academy of Sciences of the United States of America, vol. 92, no. 7, pp. 2785-2789, 1995.

[29] A. Irintchev, M. Zeschnigk, A. Starzinski-Powitz, and A. Wernig, "Expression pattern of M-cadherin in normal, denervated, and regenerating mouse muscles," Developmental Dynamics, vol. 199, no. 4, pp. 326-337, 1994.

[30] Y. Halaas, D. Duncan, J. Bernardy, P. Ondrackova, and I. Dinev, "Activation of skeletal muscle satellite cells by a device simultaneously applying high-intensity focused electromagnetic technology and novel RF technology: fluorescent microscopy facilitated detection of NCAM/CD56," Aesthetic Surgery Journal, vol. 41, no. 7, pp. NP939-NP947, 2021.

[31] C. Chu, Y. Gao, X. Lan, A. Thomas, and S. Li, "NCAM mimetic peptides: potential therapeutic target for neurological disorders," Neurochemical Research, vol. 43, no. 9, pp. 1714-1722, 2018.

[32] V. Sytnyk, I. Leshchyns'ka, and M. Schachner, "Neural cell adhesion molecules of the immunoglobulin superfamily regulate synapse formation, maintenance, and function," Trends in Neurosciences, vol. 40, no. 5, pp. 295-308, 2017.

[33] B. R. Jesudas, H. Nandeesha, V. Menon, and P. Allimuthu, "Relationship of elevated neural cell adhesion molecule 1 with interleukin-10 and disease severity in bipolar disorder," Asian Journal of Psychiatry, vol. 47, article 101849, 2020.

[34] G. Kochlamazashvili, O. Senkov, S. Grebenyuk et al., "Neural cell adhesion molecule-associated polysialic acid regulates synaptic plasticity and learning by restraining the signaling through GluN2B-containing NMDA receptors," The Journal of neuroscience : the official journal of the Society for Neuroscience, vol. 30, no. 11, pp. 4171-4183, 2010.

[35] V. Vukojevic, P. Mastrandreas, A. Arnold et al., "Evolutionary conserved role of neural cell adhesion molecule-1 in memory," Translational Psychiatry, vol. 10, no. 1, p. 217, 2020.

[36] B. Klementiev, T. Novikova, V. Novitskaya et al., "A neural cell adhesion molecule-derived peptide reduces neuropathological signs and cognitive impairment induced by $\mathrm{A} \beta_{25-35}$," Neuroscience, vol. 145, no. 1, pp. 209-224, 2007.

[37] L. Todaro, L. Puricelli, H. Gioseffi et al., "Neural cell adhesion molecule in human serum. Increased levels in dementia of the Alzheimer type," Neurobiology of Disease, vol. 15, no. 2, pp. 387-393, 2004. 